University of Nebraska - Lincoln

DigitalCommons@University of Nebraska - Lincoln

2012

Optimal control modification for robust adaptive control with large adaptive gain

Nhan T. Nguyen

NASA Ames Research Center, Nhan.T.Nguyen@nasa.gov

Follow this and additional works at: https://digitalcommons.unl.edu/nasapub

Part of the Physical Sciences and Mathematics Commons

Nguyen, Nhan T., "Optimal control modification for robust adaptive control with large adaptive gain" (2012). NASA Publications. 73.

https://digitalcommons.unl.edu/nasapub/73

This Article is brought to you for free and open access by the National Aeronautics and Space Administration at DigitalCommons@University of Nebraska - Lincoln. It has been accepted for inclusion in NASA Publications by an authorized administrator of DigitalCommons@University of Nebraska - Lincoln. 


\title{
Optimal control modification for robust adaptive control with large adaptive gain
}

\author{
Nhan T. Nguyen \\ NASA Ames Research Center, Moffett Field, CA 94035, United States
}

\section{A R T I C L E I N F O}

\section{Article history:}

Received 21 April 2011

Received in revised form

12 December 2011

Accepted 23 January 2012

Available online 22 March 2012

\section{Keywords:}

Adaptive control

Optimal control

Flight control

\begin{abstract}
A B S T R A C T
In the presence of large uncertainties, a control system needs to be able to adapt rapidly to regain performance. Fast adaptation is referred to the implementation of adaptive control with a large adaptive gain so as to reduce the tracking error rapidly. However, a large adaptive gain can lead to highfrequency oscillations which can adversely affect robustness. A new adaptive law, called optimal control modification, is presented that can achieve robust adaptation with a large adaptive gain without incurring high-frequency oscillations. The modification is based on a minimization of the $\mathcal{L}_{2}$ norm of the tracking error bounded away from some lower bound, formulated as an optimal control problem. The optimality condition is used to derive the modification based on the Pontryagin's Minimum Principle. The optimal control modification is shown to improve robustness of the standard MRAC without significantly compromising the tracking performance. Flight control simulations demonstrate the effectiveness of the new adaptive law. A series of recent, successful flight tests of this adaptive law on a NASA F/A-18A aircraft at NASA Dryden Flight Research Center further demonstrate the effectiveness of the optimal control modification adaptive law.
\end{abstract}

Published by Elsevier B.V.

\section{Introduction}

Adaptive control is a potentially promising technology that can improve a control system performance over a conventional fixedgain controller. In recent years, adaptive control has been receiving a significant amount of attention. In aerospace applications, adaptive control has been demonstrated in a number of flight vehicles. For example, NASA conducted in the last decade a flight test program of a neural net intelligent flight control system on board a modified F-15 test aircraft [1]. The ability to accommodate system uncertainties and to improve fault tolerance of a control system is a major selling point of adaptive control since conventional gain-scheduled or fixed-gain control methods are viewed as being less capable of handling off-nominal conditions caused by faults and or failures. Nonetheless, these conventional control methods tend to be robust to disturbances and unmodeled dynamics when operated as intended.

In spite of the advances made in the field of adaptive control, there exist several challenges related to the implementation of adaptive control technology in safety-critical systems. Verification and validation of adaptive control remains a major hurdle to overcome [2]. This hurdle can be traced to the lack of performance and stability metrics for adaptive control which poses a major design challenge for adaptive control. Stability robustness of adaptive control is also a major overriding concern. The fact that

E-mail address: Nhan.T.Nguyen@nasa.gov. adaptive control is nonlinear certainly makes it inherently much more difficult to ascertain stability robustness.

Even as adaptive control has been used with limited success in some applications, the possibility of high-gain control due to fast adaptation can be an issue. In certain applications, fast adaptation is needed in order to improve the tracking performance rapidly when a system is degraded significantly due to a plant damage or failure that causes large changes in system dynamics. In these situations, a large adaptive gain can be used to reduce the tracking error rapidly. However, there typically exists a balance between stability and adaptation. Fast adaptation leads to an improved tracking performance, but by the same token can also result in poor robustness that could adversely affect stability of a control system [3]. Some recent adaptive control methods have addressed fast adaptation and high-gain control, such as the $\mathscr{L}_{1}$ adaptive control [4] and the hybrid direct-indirect adaptive control [5], as well as other techniques. In the $\mathscr{L}_{1}$ approach, the implementation of a low-pass filter on the adaptive control signal effectively suppresses any high frequency oscillations that may occur due to fast adaptation. In the limit, the $\mathscr{L}_{1}$ method provides a time delay margin bounded away from zero. In the hybrid approach, direct and indirect adaptive control are blended together within the same control architecture. The indirect adaptive law uses a recursive least-squares method to adjust parameters of a controller to reduce the modeling error, and the direct adaptive law then handles any residual tracking error using a smaller adaptive gain.

To increase stability robustness of MRAC due to fast adaptation, robust modification adaptive laws can also be used. Two wellknown robust modification adaptive laws that have been used 
extensively in adaptive control are the $\sigma$ modification [6] and $\varepsilon$ modification [7]. Robust modification effectively introduces a damping mechanism into an adaptive law so as to prevent adaptive parameter bursting phenomena due to lack of robustness [3]. This paper introduces a new adaptive law based on an optimal control formulation that minimizes the $\mathscr{L}_{2}$ norm of the tracking error bounded away from some lower bound [8]. The new adaptive law, referred to as optimal control modification, can enable fast adaptation without loss of robustness. The analysis shows that the optimal control modification adaptive law can improve stability robustness of adaptive control to unmodeled dynamics. Simulations and flight testing have been conducted with this new adaptive law. The results support the effectiveness of the optimal control modification adaptive law.

\section{Optimal control modification}

Given a nonlinear plant as

$\dot{x}(t)=A x(t)+B[u(t)+f(x(t))]$

where $x(t):[0, \infty) \rightarrow \mathbb{R}^{n}$ is a state vector, $u(t):[0, \infty) \rightarrow \mathbb{R}^{p}$ is a control vector, $A \in \mathbb{R}^{n \times n}$ and $B \in \mathbb{R}^{n \times p}$ are known such that the pair $(A, B)$ is controllable, and $f(x(t)): \mathbb{R}^{n} \rightarrow \mathbb{R}^{p}$ is a matched uncertainty.

Assumption. The uncertainty $f(x(t))$ can be linearly parametrized as

$$
\begin{aligned}
f(x(t)) & =\sum_{i=1}^{m} \theta_{i}^{*} \phi_{i}(x(t))+\varepsilon(x(t)) \\
& =\Theta^{* \top} \Phi(x(t))+\varepsilon(x(t))
\end{aligned}
$$

where $\Theta^{*} \in \mathbb{R}^{m \times p}$ is an unknown constant ideal weight matrix that represents a parametric uncertainty, $\Phi(x(t)): \mathbb{R}^{n} \rightarrow \mathbb{R}^{m}$ is a vector of known bounded basis functions or regressors that are continuous and differentiable in $x$, and $\varepsilon(x(t)): \mathbb{R}^{n} \rightarrow \mathbb{R}^{p}$ is an approximation error.

The set of basis functions $\Phi(x(t))$ is chosen such that the approximation error $\varepsilon(x(t))$ is small on a compact domain $x(t) \in \mathbb{R}^{n}$. There are several function approximation methods that can be used for selecting suitable basis functions. For example, the universal approximation theorem for sigmoidal neural networks by Cybenko can be used for selecting sigmoidal basis functions $\Phi(x(t))$ [9]. Similarly, the Micchelli's theorem provides a theoretical basis for a neural net design using radial basis functions to keep the approximation error $\varepsilon(x(t))$ small [10]. Other function approximation methods such as Chebyshev orthogonal polynomials have also been used [11].

The feedback controller $u(t)$ is specified by

$u(t)=-K_{x} x(t)+K_{r} r(t)-u_{a d}(t)$

where $r(t):[0, \infty) \rightarrow \mathbb{R}^{p} \in \mathscr{L}_{\infty}$ is a command vector, $K_{x} \in \mathbb{R}^{p \times n}$ is a stable gain matrix such that $A-B K_{x}$ is Hurwitz, $K_{r} \in \mathbb{R}^{p \times p}$ is a gain matrix for $r(t)$, and $u_{a d}(t) \in \mathbb{R}^{p}$ is a direct adaptive signal which estimates the parametric uncertainty in the plant such that

$u_{a d}(t)=\Theta^{\top}(t) \Phi(x(t))$

where $\Theta(t) \in \mathbb{R}^{m \times p}$ is an estimate of the parametric uncertainty $\Theta^{*}$.

Then, the reference model is specified as

$\dot{x}_{m}(t)=A_{m} x_{m}(t)+B_{m} r(t)$

where $A_{m} \in \mathbb{R}^{n \times n}$ and $B_{m} \in \mathbb{R}^{n \times p}$ are given by

$A_{m}=A-B K_{x}$

$B_{m}=B K_{r}$.
Let $\tilde{\Theta}(t)=\Theta(t)-\Theta^{*}$ be an estimation error of the parametric uncertainty and define the tracking error as $e(t)=x_{m}(t)-x(t)$ then the tracking error equation becomes

$\dot{e}(t)=A_{m} e(t)+B\left[\tilde{\Theta}^{\top}(t) \Phi(x(t))-\varepsilon(x(t))\right]$.

Proposition. The following optimal control modification adaptive law

$\dot{\Theta}(t)=-\Gamma \Phi(x(t))\left[e^{\top}(t) P-v \Phi^{\top}(x(t)) \Theta(t) B^{\top} P A_{m}^{-1}\right] B$

provides an update law that minimizes $\|e(t)\|_{\mathscr{L}_{2}}$ associated with an infinite-time horizon cost function

$J=\lim _{t \rightarrow \infty} \frac{1}{2} \int_{0}^{t_{f}}[e(t)-\Delta(t)]^{\top} Q[e(t)-\Delta(t)] \mathrm{d} t$

subject to Eq. (8), where $\Delta$ represents the unknown lower bound of the tracking error, $Q=Q^{\top}>0 \in \mathbb{R}^{n \times n}$ is a weighting matrix, $\Gamma=\Gamma^{\top}>0 \in \mathbb{R}^{m \times m}$ is an adaptive gain matrix, $v>0 \in \mathbb{R}$ is a modification parameter, and $P=P^{\top}>0 \in \mathbb{R}^{n \times n}$ solves

$P A_{m}+A_{m}^{\top} P=-Q$.

Proof. The cost function $J$ is convex and represents the distance measured from a point on the trajectory of $e(t)$ to the normal surface of a hypersphere $B_{\Delta}=\left\{e(t) \in \mathbb{R}^{n}:\|e(t)\| \leq\|\Delta(t)\|\right\} \subset$ $\mathscr{D} \subset \mathbb{R}^{n}$. The cost function is designed to provide stability robustness by not seeking an asymptotic tracking error that tends to zero as $t_{f} \rightarrow \infty$, but rather one that tends to some lower bound away from the origin. By not requiring an asymptotic tracking error, the adaptation can be made more robust. Therefore, the tracking performance can be traded with stability robustness by a suitable selection of the modification parameter $v$. Increasing the tracking performance by reducing $v$ will decrease stability robustness of the adaptive law to unmodeled dynamics and vice versa.

This optimal control problem can be formulated by the Pontryagin's Minimum Principle. Defining a Hamiltonian

$$
\begin{gathered}
H(e(t), \tilde{\Theta}(t))=\frac{1}{2}[e(t)-\Delta(t)]^{\top} Q[e(t)-\Delta(t)] \\
+p^{\top}(t)\left[A_{m} e(t)+B \tilde{\Theta}^{\top}(t) \Phi(x(t))-B \varepsilon(x(t))\right]
\end{gathered}
$$

where $p(t):[0, \infty) \rightarrow \mathbb{R}^{n}$ is an adjoint variable, then the necessary condition gives

$\dot{p}(t)=-\nabla H_{e}^{\top}=-Q[e(t)-\Delta(t)]-A_{m}^{\top} p(t)$

with the transversality condition $p\left(t_{f} \rightarrow \infty\right)=0$ since $e(0)$ is known. Treating $\tilde{\Theta}^{\top}(t) \Phi(x)$ as a control variable, then the optimality condition is obtained by

$\nabla H_{\tilde{\Theta}^{\top} \Phi}=p^{\top}(t) B$.

The adaptive law can then be formulated by a gradient update law as

$$
\begin{aligned}
\dot{\tilde{\Theta}}(t) & =-\Gamma \nabla H_{\tilde{\Theta}^{\top}}=-\Gamma \Phi(x(t)) \nabla H_{\tilde{\Theta}^{\top} \Phi} \\
& =-\Gamma \Phi(x(t)) p^{\top}(t) B .
\end{aligned}
$$

An "approximate" solution of $p(t)$ can be obtained using a "sweep" method [12] by letting $p(t)=W(t) e(t)+$ $R(t) \Theta^{\top}(t) \Phi(x(t))$ where $W(t):[0, \infty) \rightarrow \mathbb{R}^{n \times n}$ and $R(t):$ 
$[0, \infty) \rightarrow \mathbb{R}^{n \times p}$ are matrices of influence functions [12]. Then

$$
\begin{aligned}
\dot{W}(t) & e(t)+W(t)\left[A_{m} e(t)+B \Theta^{\top}(t) \Phi(x(t))\right. \\
& \left.-B \Theta^{* \top} \Phi(x(t))-B \varepsilon(x(t))\right]+\dot{R}(t) \Theta^{\top}(t) \Phi(x(t)) \\
& +R(t) \frac{\mathrm{d}\left(\Theta^{\top}(t) \Phi(x(t))\right)}{\mathrm{d} t}=-Q[e(t)-\Delta(t)] \\
& -A_{m}^{\top}\left[W(t) e(t)+R(t) \Theta^{\top}(t) \Phi(x(t))\right]
\end{aligned}
$$

which yields the following equations

$$
\begin{aligned}
& \dot{W}(t)+W(t) A_{m}+A_{m}^{\top} W(t)+Q=0 \\
& \dot{R}(t)+W(t) B+A_{m}^{\top} R(t)=0 \\
& Q \Delta(t)-R(t) \frac{\mathrm{d}\left(\Theta^{\top}(t) \Phi(x(t))\right)}{\mathrm{d} t} \\
& \quad+W(t) B\left[\Theta^{* \top} \Phi(x(t))+\varepsilon(x(t))\right]=0
\end{aligned}
$$

subject to the transversality conditions $W\left(t_{f} \rightarrow \infty\right)=0$ and $R\left(t_{f} \rightarrow \infty\right)=0$.

The existence and uniqueness of the solution of the Lyapunov differential equation (17) is well-established [13]. It follows that Eq. (18) also has a stable, unique solution in time-to-go $\tau=t_{f}-t$. Then

$$
\begin{aligned}
& -\frac{\mathrm{d} W(\tau)}{\mathrm{d} \tau}+W(\tau) A_{m}+A_{m}^{\top} W(\tau)+Q=0 \\
& -\frac{\mathrm{d} R(\tau)}{\mathrm{d} \tau}+W(\tau) B+A_{m}^{\top} R(\tau)=0
\end{aligned}
$$

subject to the initial conditions in time-to-go $W(0)=0$ and $R(0)=0$.

The infinite-time horizon solutions of $W(\tau)$ and $R(\tau)$ then tend to their constant solutions. That is, $W(\tau) \rightarrow P$ and $R(\tau) \rightarrow S$ as $\tau \rightarrow \infty$ where

$P A_{m}+A_{m}^{\top} P=-Q$

$S=-A_{m}^{-\top} P B$.

The adjoint $p$ is then obtained as

$p(t)=P e(t)-A_{m}^{-\top} P B \Theta^{\top}(t) \Phi(x(t))$. follows:

Thus the optimal solution provides a unique adaptive law as

$$
\dot{\Theta}(t)=-\Gamma \Phi(x(t))\left[e^{\top}(t) P-\Phi^{\top}(x(t)) \Theta(t) B^{\top} P A_{m}^{-1}\right] B .
$$

However, in many instances, sub-optimal solutions may provide a more flexible, yet practical approach to a control design by allowing a trade-off between the optimality and other design considerations. In the present approach, the optimal solution ensures optimal stability robustness of the optimal control modification adaptive law. As with any control design, performance and robustness are often considered as two competing design requirements. Increasing robustness tends to require a compromise in performance and vice versa. Thus, to enable the optimal control modification adaptive law to be sufficiently flexible for a control design, the modification parameter $v>0$ is introduced as a gain to allow for adjustments of the modification term.

The role of the modification parameter $v$ is important. If tracking performance is more desired in a control design than stability robustness, then $v$ could be selected to be a small value. In the limit when $v=0$, the standard MRAC is recovered and asymptotic tracking performance is achieved but at the expense of stability robustness. On the other hand, if stability robustness is a priority in a design, then a larger value of $v$ should be chosen.
Thus, the solution of $S$ is modified as

$S=-v A_{m}^{-\top} P B$.

The adjoint $p$ is then obtained as

$p(t)=P e(t)-v A_{m}^{-\top} P B \Theta^{\top}(t) \Phi(x(t))$.

Since $\Theta^{*}$ is a constant ideal weight matrix, then it follows that the adaptive law (9) is obtained from Eqs. (15) and (27).

The bound on $\Delta(t)$ as $t_{f} \rightarrow \infty$ can be estimated by

$$
\begin{aligned}
\|\Delta(t)\| \leq & \left\|Q^{-1}\right\|\left[v\left\|A_{m}^{-\top} P B\right\|\left\|\frac{\mathrm{d}\left(\Theta^{\top}(t) \Phi(x(t))\right)}{\mathrm{d} t}\right\|\right. \\
& +\|P B\|\|f(x(t))\|]
\end{aligned}
$$

which is dependent upon the modification parameter $v$ and the matched uncertainty $\|f(x(t))\|$.

The optimal control formulation shows that $\|\Delta(t)\|$ will always remain finite as long as the uncertainty exists. Therefore, the optimal control modification will always result in a bounded tracking error. In contrast, the standard MRAC can achieve asymptotic tracking error, but at the expense of stability robustness to unmodeled dynamics. The lack of stability robustness of the standard MRAC is well-known [14]. In many real systems, asymptotic tracking is a very demanding requirement that usually cannot easily be met without any restrictions. The optimal control formulation therefore demonstrates that bounded tracking is a more realistic control objective if stability robustness is to be achieved concurrently. Since $\|\Delta(t)\|$ is proportional to the modification parameter $v$, the optimal control modification adaptive law can be designed judiciously to trade tracking performance with stability robustness. Increasing the value of the modification parameter $v$ will reduce tracking performance but by the same token increase stability robustness. This trade-off exists in all robust modification schemes such as the $\sigma$ modification or $\varepsilon$ modification.

Theorem. The adaptive law (9) results in stable and ultimately bounded tracking error $e(t)$ with an ultimate bound

$\rho=\sqrt{\frac{\lambda_{\max }(P) r^{2}+\lambda_{\max }\left(\Gamma^{-1}\right) \kappa^{2}}{\lambda_{\min }(P)}}$

where $r=c_{2}+\sqrt{c_{5} / c_{1}}$ and $\kappa=c_{4}+\sqrt{c_{5} / c_{3}}$ for some positive real numbers $c_{1} \triangleq \lambda_{\min }(Q), c_{2} \triangleq\|P B\| \varepsilon_{0} / \lambda_{\min }(Q)$, $c_{3} \triangleq v\|\Phi(x(t))\|^{2} \lambda_{\text {min }}\left(B^{\top} A_{m}^{-\top} Q A_{m}^{-1} B\right), c_{4} \triangleq\left\|B^{\top} P A_{m}^{-1} B\right\| \Theta_{0}^{*} / \lambda_{\text {min }}$ $\left(B^{\top} A_{m}^{-\top} Q A_{m}^{-1} B\right)$, and $c_{5} \triangleq c_{1} c_{2}^{2}+c_{3} c_{4}^{2}$ with $\varepsilon_{0}=\sup _{x(t) \in \mathcal{D}}\|\varepsilon(x(t))\|$ and $\Theta_{0}^{*}=\left\|\Theta^{*}\right\|$.

Proof. Choose a Lyapunov candidate function

$V(t)=e^{\top}(t) P e(t)+\operatorname{trace}\left(\tilde{\Theta}^{\top}(t) \Gamma^{-1} \tilde{\Theta}(t)\right)$.

Evaluating $\dot{V}$ yields

$$
\begin{aligned}
\dot{V}(t)= & e^{\top}(t)\left(A_{m} P+P A_{m}\right) e(t) \\
& +2 e^{\top}(t) P B\left[\tilde{\Theta}^{\top}(t) \Phi(x(t))-\varepsilon(x(t))\right] \\
& -2 \operatorname{trace}\left(\tilde { \Theta } ^ { \top } ( t ) \Phi ( x ( t ) ) \left[e^{\top}(t) P B\right.\right. \\
& \left.\left.-v \Phi^{\top}(x(t)) \Theta(t) B^{\top} P A_{m}^{-1} B\right]\right) .
\end{aligned}
$$

For any arbitrary row vectors $A=\left[\begin{array}{lllll}a_{1} & \cdots & a_{i} & \cdots & a_{n}\end{array}\right]$ $\in \mathbb{R}^{n}$ and $B=\left[\begin{array}{lllll}b_{1} & \cdots & b_{i} & \cdots & b_{n}\end{array}\right] \in \mathbb{R}^{n}$, trace $\left(A^{\top} B\right)=$ 
$B A^{\top}=\sum_{i=1}^{n} a_{i} b_{i}$. Therefore, $\dot{V}(t)$ can be written as

$$
\begin{aligned}
\dot{V}(t)= & -e^{\top}(t) Q e(t)+2 e^{\top}(t) P B\left[\tilde{\Theta}^{\top}(t) \Phi(x(t))-\varepsilon(x(t))\right] \\
& -2 e^{\top}(t) P B \tilde{\Theta}^{\top}(t) \Phi(x(t)) \\
& +2 \nu \Phi^{\top}(x(t)) \Theta(t) B^{\top} P A_{m}^{-1} B \tilde{\Theta}^{\top}(t) \Phi(x(t)) .
\end{aligned}
$$

The sign-definiteness of the term $P A_{m}^{-1}$ is now considered. Recall that a general real matrix $G$ is positive (negative) definite if and only if its symmetric part $G=\frac{1}{2}\left(G+G^{\top}\right)$ is also positive (negative) definite. Then, by pre- and post-multiplication of Eq. (11) by $A_{m}^{-\top}$ and $A_{m}^{-1}$, respectively, $P A_{m}^{-1}$ can be decomposed into a symmetric part $M$ and anti-symmetric part $N$ as

$P A_{m}^{-1}=M+N$

where

$M=\frac{1}{2}\left(A_{m}^{-\top} P+P A_{m}^{-1}\right)=-\frac{1}{2} A_{m}^{-\top} Q A_{m}^{-1}$

$N=\frac{1}{2}\left(P A_{m}^{-1}-A_{m}^{-\top} P\right)$.

Since the symmetric part $M<0$, then $P A_{m}^{-1}<0$. Thus, $\dot{V}(t)$ becomes

$$
\begin{aligned}
\dot{V}(t)= & -e^{\top}(t) Q e(t)-2 e^{\top}(t) P B \varepsilon(x(t)) \\
& -v \Phi^{\top}(x(t)) \tilde{\Theta}(t) B^{\top} A_{m}^{-\top} Q A_{m}^{-1} B \tilde{\Theta}^{\top}(t) \Phi(x(t)) \\
& +2 v \Phi^{\top}(x(t)) \tilde{\Theta}(t) B^{\top} N B \tilde{\Theta}^{\top}(t) \Phi(x(t)) \\
& +2 v \Phi^{\top}(x(t)) \Theta_{0}^{*} B^{\top} P A_{m}^{-1} B \tilde{\Theta}^{\top}(t) \Phi(x(t)) .
\end{aligned}
$$

Letting $y(t)=B \tilde{\Theta}^{\top}(t) \Phi(x(t))$ and using the property $y^{\top}(t)$ $N y(t)=0$ for an anti-symmetric matrix $N, \dot{V}(t)$ is reduced to

$$
\begin{aligned}
\dot{V}(t)= & -e^{\top}(t) Q e(t)-2 e^{\top}(t) P B \varepsilon(x(t)) \\
& -v \Phi^{\top}(x(t)) \tilde{\Theta}(t) B^{\top} A_{m}^{-\top} Q A_{m}^{-1} B \tilde{\Theta}^{\top}(t) \Phi(x(t)) \\
& +2 v \Phi^{\top}(x(t)) \Theta_{0}^{*} B^{\top} P A_{m}^{-1} B \tilde{\Theta}^{\top}(t) \Phi(x(t))
\end{aligned}
$$

which is bounded by

$$
\begin{aligned}
\dot{V}(t) \leq & -\|e(t)\|\left[\lambda_{\min }(Q)\|e(t)\|-2\|P B\| \varepsilon_{0}\right] \\
& -v\|\Phi(x(t))\|^{2}\|\tilde{\Theta}(t)\|\left[\lambda_{\min }\left(B^{\top} A_{m}^{-\top} Q A_{m}^{-1} B\right)\right. \\
& \left.\times\|\tilde{\Theta}(t)\|-2\left\|B^{\top} P A_{m}^{-1} B\right\| \Theta_{0}^{*}\right] .
\end{aligned}
$$

By completing the squares, $\dot{V}(t)$ is expressed as

$\dot{V}(t) \leq-c_{1}\left[\|e(t)\|-c_{2}\right]^{2}-c_{3}\left[\|\tilde{\Theta}(t)\|-c_{4}\right]^{2}+c_{5}$

where $c_{1} \triangleq \lambda_{\min }(Q), c_{2} \triangleq\|P B\| \varepsilon_{0} / \lambda_{\min }(Q), c_{3} \triangleq v\|\Phi(x(t))\|^{2}$ $\lambda_{\min }\left(B^{\top} A_{m}^{-\top} Q A_{m}^{-1} B\right), c_{4} \triangleq\left\|B^{\top} P A_{m}^{-1} B\right\| \Theta_{0}^{*} / \lambda_{\min }\left(B^{\top} A_{m}^{-\top} Q A_{m}^{-1} B\right)$, and $c_{5} \triangleq c_{1} c_{2}^{2}+c_{3} c_{4}^{2}$ are all positive real numbers.

Let

$$
\begin{aligned}
B_{r}= & \left\{(e(t), \tilde{\Theta}(t)) \in \mathbb{R}^{n} \times \mathbb{R}^{m \times p}: c_{1}\left[\|e(t)\|-c_{2}\right]^{2}\right. \\
& \left.+c_{3}\left[\|\tilde{\Theta}(t)\|-c_{4}\right]^{2} \leq c_{5}\right\} .
\end{aligned}
$$

Then $\dot{V}>0$ inside of $B_{r}$ but $\dot{V} \leq 0$ outside of $B_{r}$, therefore $V$ is a decreasing function outside of $B_{r}$. The trajectory $(e(0), \tilde{\Theta}(0))$ will remain bounded at all times and $e(t)$ will be ultimately bounded after some time $t>T$ by an ultimate bound

$\rho=\sqrt{\frac{\lambda_{\text {max }}(P) r^{2}+\lambda_{\text {max }}\left(\Gamma^{-1}\right) \kappa^{2}}{\lambda_{\text {min }}(P)}}$

where $r=c_{2}+\sqrt{c_{5} / c_{1}}$ and $\kappa=c_{4}+\sqrt{c_{5} / c_{3}}$.
Table 1

Robust modifications to MRAC

\begin{tabular}{ll}
\hline Modification & Adaptive law \\
\hline$\sigma$ & $\dot{\Theta}=-\Gamma\left[\Phi(x) e^{\top} P B+\sigma \Theta\right], \sigma>0$ \\
$\varepsilon$ & $\dot{\Theta}=-\Gamma\left[\Phi(x) e^{\top} P B+\mu\left\|e^{\top} P B\right\| \Theta\right], \mu>0$ \\
$\begin{array}{l}\text { Optimal } \\
\text { control }\end{array}$ & $\dot{\Theta}=-\Gamma\left[\Phi(x) e^{\top} P B-\nu \Phi(x) \Phi^{\top}(x) \Theta B^{\top} P A_{m}^{-1} B\right], \nu>0$ \\
$\begin{array}{l}\text { Adaptive loop } \\
\text { recovery }\end{array}$ & $\dot{\Theta}=-\Gamma\left[\Phi(x) e^{\top} P B+\eta \frac{\mathrm{d} \Phi(x)}{\mathrm{d} x} \frac{\mathrm{d} \Phi^{\top}(x)}{\mathrm{d} x} \Theta\right], \eta>0$
\end{tabular}

It is noted that if $v=0$ which recovers the standard MRAC and if $\varepsilon_{0}=0$, then it can be shown by the Barbalat's lemma that $\dot{V}(t)$ is uniformly continuous and $e(t) \rightarrow 0$ as $t \rightarrow \infty$ [15].

Remark 1. Utilizing $P A_{m}^{-1}=M+N$, an alternative expression for the optimal control modification adaptive law is given by

$$
\begin{aligned}
\dot{\Theta}(t)= & -\Gamma \Phi(x(t))\left[e^{\top}(t) P\right. \\
& \left.+\frac{v}{2} \Phi^{\top}(x(t)) \Theta(t) B^{\top} A_{m}^{-\top} Q A_{m}^{-1}\right] B .
\end{aligned}
$$

Remark 2. The effect of the optimal control modification is to add damping to the weight update law so as to reduce high-frequency oscillations in the weights. This scheme is contrasted to the wellknown $\sigma$ modification [6] and $\varepsilon$ modification [7] which add a constant damping term as in the case of the $\sigma$ modification and a damping term proportional to $\|e(t)\|$ as in the case of the $\varepsilon$ modification. A recent modification, called adaptive loop recovery, provides a damping term proportional to $\frac{\mathrm{d} \Phi(x(t))}{\mathrm{d} x} \frac{\mathrm{d} \Phi^{\top}(x(t))}{\mathrm{d} x}$ [16]. These adaptive laws are compared (see Table 1 ).

Remark 3. The transient performance of the optimal control modification is dependent upon the modification parameter $v$ and the adaptive gain $\Gamma$. This is similar to the transient performance of the $\sigma$ modification and $\varepsilon$ modification which is dependent upon the modification parameters $\sigma$ and $\mu$. Increasing the values of the modification parameters $\nu, \sigma$, and $\mu$ in all these robust modification adaptive laws will degrade the transient performance. However, with the optimal control modification, increasing $v$ also increases stability robustness which allows a large adaptive gain to be used to improve the transient performance. Thus, for the optimal control modification, the net effect is that both the transient performance and stability robustness can be improved simultaneously using a large adaptive gain and a properly chosen modification parameter $v$.

The issue of the lack of robustness of model-reference adaptive control is well understood [14]. As the adaptive gain $\Gamma$ increases, the tracking performance of the standard MRAC adaptive law improves, but at the same time robustness against time delay and or unmodeled dynamics also suffers. In theory, as the adaptive gain $\Gamma$ tends to infinity, the time delay margin which is a measure of robustness tends to zero. Thus, if the uncertainty is large and requires fast adaptation; i.e., a large adaptive gain, the standard MRAC will not be able to maintain sufficient robustness to ensure that the adaptive parameters will remain bounded. In contrast, the optimal control modification can be shown to maintain a measure of robustness for fast adaptation.

Consider an asymptotic solution of the optimal control modification adaptive law when $\Gamma \rightarrow \infty$ for a linear match uncertainty; i.e., $f(x(t))=\Theta^{* \top} x(t)$. Then it can be seen that

$B \Theta^{\top}(t) \Phi(x(t)) \rightarrow \frac{1}{v} P^{-1} A_{m}^{\top} P e(t)$. 


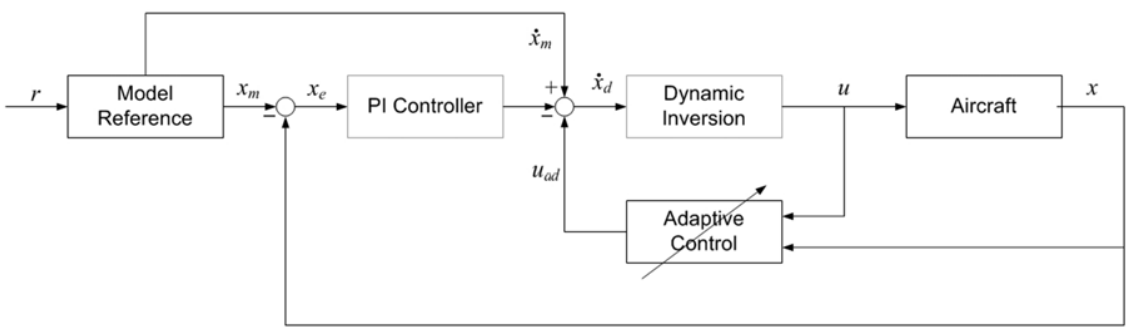

Fig. 1. Adaptive flight control architecture.

The closed-loop tracking error equation in the limit then becomes

$\dot{e}(t)=\left(A_{m}+\frac{1}{v} P^{-1} A_{m}^{\top} P+B \Theta^{* \top}\right) e(t)-B \Theta^{* \top} x_{m}(t)$

which interestingly is a linear time invariant (LTI) system.

Thus, the optimal control modification adaptive law can be designed by a suitable selection of the modification parameter $v$ to guarantee stability robustness for a given a priori specification of the parametric uncertainty $\Theta^{*}$ under the condition of fast adaptation. Since the closed-loop tracking error in the limit is LTI, stability margins like phase and time delay margins of the optimal control modification can easily be computed using many available linear stability analysis tool. These margins will remain finite and positive even as the adaptive gain $\Gamma$ tends to a large value. This limiting behavior is very similar to that of the $\mathscr{L}_{1}$ adaptive control [4].

\section{Flight control application}

Adaptive flight control can be used to provide consistent handling qualities and restore stability of aircraft under offnominal flight conditions due to failures or damage. Consider the following inner loop adaptive flight control architecture as shown in Fig. 1. The control architecture comprises: (1) a reference model that translates rate commands into desired acceleration commands, (2) a proportional-integral (PI) feedback control for rate stabilization and tracking, (3) a dynamic inversion controller that computes actuator commands using desired acceleration commands, and (4) a direct MRAC augmentation which can be the standard MRAC, $\varepsilon$ modification, or the optimal control modification.

The equations of motion are expressed as

$$
\begin{aligned}
& \dot{x}(t)=A_{11} x(t)+A_{12} z(t)+B_{1} u(t)+f_{1}(x(t), z(t)) \\
& \dot{z}(t)=A_{21} x(t)+A_{22} z(t)+B_{2} u(t)+f_{2}(x(t), z(t))
\end{aligned}
$$

where $A_{i j}$ and $B_{i}, i=1,2, j=1,2$ are nominal undamaged plant matrices which are assumed to be known, $x(t)=$ $[p(t) q(t) r(t)]^{\top}$ is an inner loop vector of roll, pitch, and yaw rates; $z(t)=[\Delta \phi(t) \Delta \alpha(t) \Delta \beta(t) \Delta V(t) \Delta h(t) \Delta \theta(t)]^{\top}$ is an outer loop vector of perturbation in the bank angle $\Delta \phi(t)$, angle of attack $\Delta \alpha(t)$, sideslip angle $\Delta \beta(t)$, airspeed $\Delta V(t)$, altitude $\Delta h(t)$, and pitch angle $\Delta \theta(t) ; u(t)=\left[\Delta \delta_{a}(t) \Delta \delta_{e}(t) \Delta \delta_{r}(t)\right]^{\top}$ is a vector of incremental aileron, elevator, and rudder deflections; and $f_{i}(x(t), z(t)), i=1,2$ is an uncertainty due to damage which can be approximated as

$f_{i}(x(t), z(t))=\Theta_{i}^{* \top} \Phi(x(t), z(t))+\varepsilon(x(t), z(t))$.

The inner loop rate feedback control is designed to improve aircraft rate response characteristics such as the short period mode and the dutch roll mode. The outer loop variables generally have a slower response time. A second-order reference model is specified to provide desired handling qualities with good damping and natural frequency characteristics. Let $p_{m}(t), q_{m}(t)$, and $r_{m}(t)$ be the reference roll, pitch, and yaw rates. Then the reference model can be represented as

$\dot{x}_{m}(t)=-K_{p} x_{m}(t)-K_{i} \int_{0}^{t} x_{m}(\tau) \mathrm{d} \tau+G r(t)$

where $x_{m}(t)=\left[\begin{array}{lll}p_{m}(t) & q_{m}(t) & r_{m}(t)\end{array}\right]^{\top} ; K_{p}=\operatorname{diag}\left(2 \zeta_{p} \omega_{p}, 2 \zeta_{q} \omega_{q}\right.$, $\left.2 \zeta_{r} \omega_{r}\right) ; K_{i}=\operatorname{diag}\left(\omega_{p}^{2}, \omega_{q}^{2}, \omega_{r}^{2}\right) ; G=\operatorname{diag}\left(g_{p}, g_{q}, g_{r}\right) ; r(t)=$ $\left[\begin{array}{lll}\delta_{\text {lat }}(t) & \delta_{\text {lon }}(t) & \delta_{\text {rud }}(t)\end{array}\right]^{\top} ; \omega_{p}, \omega_{q}$, and $\omega_{r}$ are the desired natural frequencies in the roll, pitch, and yaw axes; $\zeta_{p}, \zeta_{q}$, and $\zeta_{r}$ are the desired damping ratios; $\delta_{\text {lat }}(t), \delta_{\text {lon }}(t)$, and $\delta_{\text {rud }}(t)$ are the pilot inputs; and $g_{p}, g_{q}$, and $g_{r}$ are input gains.

Assuming the pair $\left(A_{11}, B_{1}\right)$ is controllable and $z(t)$ is stabilizable, an angular rate feedback dynamic inversion controller is computed as

$$
\begin{aligned}
u_{c}(t)= & B_{1}^{-1}\left[-\left(K_{p}+\frac{K_{i}}{s}\right) x(t)+G r(t)-A_{11} x(t)\right. \\
& \left.-A_{12} z(t)-\Theta_{1}^{\top}(t) \Phi(x(t), z(t))\right]
\end{aligned}
$$

where $u_{c}$ is a control surface deflection command vector as an input to a flight control actuator system which can be modeled as a first-order system

$\dot{u}=-\Lambda\left(u-u_{c}\right)$

where $\Lambda=\operatorname{diag}\left(\lambda_{a}, \lambda_{e}, \lambda_{r}\right)>0$ is a vector of actuator rates for aileron, elevator, and rudder.

Let $e(t)=\left[\int_{0}^{t}\left[x_{m}(t)-x(t)\right] \mathrm{d} \tau \quad x_{m}(t)-x(t)\right]^{\top}$ be the tracking error, then

$$
\begin{aligned}
\dot{e}(t)= & A_{m} e(t)+B\left[\Theta_{1}^{\top}(t) \Phi(t) \Phi(x(t), z(t))\right. \\
& \left.-f_{1}(t) \Phi(x(t), z(t))\right]
\end{aligned}
$$

where

$A_{m}=\left[\begin{array}{cc}0 & I \\ -K_{i} & -K_{p}\end{array}\right]$

$B=\left[\begin{array}{l}0 \\ I\end{array}\right]$.

Let $Q=2 I$, then the solution of Eq. (11) yields

$P=\left[\begin{array}{cc}K_{i}^{-1} K_{p}+K_{p}^{-1}\left(K_{i}+I\right) & K_{i}^{-1} \\ K_{i}^{-1} & K_{p}^{-1}\left(I+K_{i}^{-1}\right)\end{array}\right]>0$.

$A_{m}^{-1}$ is computed to be

$A_{m}^{-1}=\left[\begin{array}{cc}-K_{i}^{-1} K_{p} & -K_{i}^{-1} \\ I & 0\end{array}\right]$ 


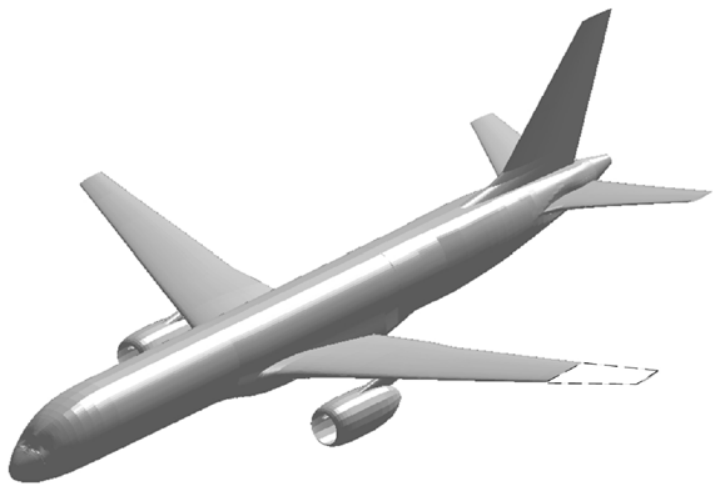

Fig. 2. Left wing damaged generic transport model.

Evaluating the term $B^{\top} P A_{m}^{-1} B$ yields

$B^{\top} P A_{m}^{-1} B=-B^{\top} A_{m}^{-\top} A_{m}^{-1} B=-K_{i}^{-2}<0$. by

The optimal control modification adaptive law (9) is then given

$$
\begin{aligned}
\dot{\Theta}_{1}(t)= & -\Gamma \Phi(x(t), z(t))\left[e^{\top}(t) P B\right. \\
& \left.+v \Phi^{\top}(x(t), z(t)) \Theta_{1}(t) K_{i}^{-2}\right] .
\end{aligned}
$$

Thus, for the PI rate control implementation, the optimal control modification term turns out to be inversely proportional to the square of the integral gain or the square of the frequency.

Remark 4. Based on Eq. (57), the optimal control modification can also be expressed as

$\dot{\Theta}(t)=-\Gamma \Phi(x(t))\left[e^{\top}(t) P B+\Phi^{\top}(x(t)) \Theta(t) G^{2}\right]$

where $G^{2}>0$ is a diagonal matrix of modification parameters with the following special cases:

- $G^{2}=\nu K_{i}^{-2}$ for first-order reference models with proportional-integral-derivative control.

- $G^{2}=\nu K_{p}^{-2}$ for first-order reference models with proportional control or second-order reference models with proportional-derivative control.

A flight simulation was conducted using a generic transport model (GTM) [17] with a $28 \%$ loss of the left wing as shown in Fig. 2. Since the damage is asymmetric, all the three axes are fully coupled together throughout flight. A level flight condition of Mach 0.6 at $15,000 \mathrm{ft}$ is selected. Upon damage, the aircraft is re-trimmed with $T=13,951 \mathrm{lb}, \bar{\alpha}=5.86^{\circ}, \bar{\phi}=-3.16^{\circ}, \bar{\delta}_{a}=27.32^{\circ}$, $\bar{\delta}_{e}=-0.53^{\circ}, \bar{\delta}_{r}=-1.26^{\circ}$. The remaining right aileron is the only roll control effector available. The reference model is specified by $\omega_{p}=2.0 \mathrm{rad} / \mathrm{s}, \omega_{q}=1.5 \mathrm{rad} / \mathrm{s}, \omega_{r}=1.0 \mathrm{rad} / \mathrm{s}$, and $\zeta_{p}=\zeta_{q}=$ $\zeta_{r}=1 / \sqrt{2}$. The actuator dynamics are modeled with $\lambda_{a}=\lambda_{e}=$ $\lambda_{r}=50 / \mathrm{s}$ and position limits of $\pm 35^{\circ}$ for the aileron and elevator and $\pm 10^{\circ}$ for the rudder.

The pilot pitch rate command is simulated with a series of ramp input longitudinal stick command doublets, corresponding to the reference pitch angle $\pm 3.81^{\circ}$ from trim. The tracking performance of the baseline controller without adaptation is compared against the standard MRAC, the $\varepsilon$ modification with $\mu=0.1$, and the optimal control modification with $v=0.1$. The adaptive gains are selected as large as possible within the numerical stability limit of the adaptive laws. This results in $\Gamma=60$ for the standard MRAC, $\Gamma=800$ for the $\varepsilon$ modification, and $\Gamma=2580$ for the optimal control modification. Thus, it can be seen that the optimal control modification can tolerate a much larger adaptive gain than the standard MRAC. This large adaptive gain allows the optimal control modification to better adapt to uncertainties than the standard MRAC.

The aircraft angular rate responses are shown in Fig. 3. The baseline controller without adaptation produces a rather poor tracking of the reference pitch rate. Both the standard MRAC and $\varepsilon$ modification improve the tracking performance significantly. The optimal control modification (OCM) is able to provide better tracking than both the standard MRAC and $\varepsilon$ modification. Due to the asymmetric wing damage, the roll axis is most affected. With the baseline controller, there is a significant roll rate of as high as $20^{\circ} / \mathrm{s}$. Both the standard MRAC and $\varepsilon$ modification reduce the maximum amplitude of the roll rate to about $10^{\circ} / \mathrm{s}$. The optimal control modification further reduces the roll rate to a maximum value of about $4^{\circ} / \mathrm{s}$. All the three adaptive controllers significantly reduce the yaw rate to a very low level. The $\varepsilon$ modification is observed to perform slightly better than the standard MRAC and optimal control modification in the yaw axis.

Fig. 4 is the plot of the tracking error $\mathscr{L}_{2}$ norm for all the three axes that compares the overall tracking performance of the four controllers. When there is no adaptation, the tracking error norm appears to grow considerably in the first $10 \mathrm{~s}$. The $\varepsilon$ modification actually results in higher tracking error norm than the standard MRAC. This is due to the poorer tracking performance in the roll rate of the $\varepsilon$ modification as compared to the standard MRAC. The optimal control modification results in the smallest tracking error norm as compared to both the standard MRAC and $\varepsilon$ modification. Thus, the optimal control modification overall performs significantly better than both the standard MRAC and $\varepsilon$ modification.

The aircraft attitude responses are shown in Fig. 5. When there is no adaptation, the pitch attitude could not be followed accurately. With adaptive control, the tracking is much improved and the optimal control modification follows the pitch command better than both the standard MRAC and $\varepsilon$ modification. Without adaptation, as expected the damaged aircraft exhibits a rather severe roll behavior with a large bank angle between $-30^{\circ}$ and $20^{\circ}$. Both the standard MRAC and $\varepsilon$ modification reduce the bank angle significantly. However, the optimal control modification shows a drastic improvement in the arrest of the roll motion with the bank angle maintained very close to the trim value. All the three adaptive controllers produce similar angle of attack responses. With the optimal control modification, the sideslip angle is reduced to near zero, while both the standard MRAC and $\varepsilon$ modification still show considerable sideslip angle responses.

The control surface deflections are plotted in Fig. 6. Because of the wing damage, the damaged aircraft has to be trimmed with a rather large aileron deflection. This causes the roll control authority to be severely limited. Therefore, a roll control saturation is present in all cases. The elevator deflection is nominally similar for all the four controllers and is well within its control authority. The rudder deflection produced by the baseline controller is quite significant. Generally, it is desired to keep the rudder deflection as small as possible in normal operation. Typically, the rudder deflection limit is reduced as the airspeed and altitude increase. Both the standard MRAC and $\varepsilon$ modification reduce the baseline rudder deflection to some extent, but the optimal control modification is observed to produce the smallest rudder deflection.

To demonstrate stability robustness of the optimal control modification, the time delay margin (TDM) is computed numerically in the simulation as a function of the parameter $v$. A time delay is introduced between the actuators and the damaged aircraft plant model and is adjusted until the optimal control modification adaptive law is on the verge of instability. The results are plotted in Fig. 7 for an adaptive gain $\Gamma=60$. As $v$ increases, the time delay margin also increases. This results in a more robust controller that can tolerate a larger time delay which acts to destabilize the 

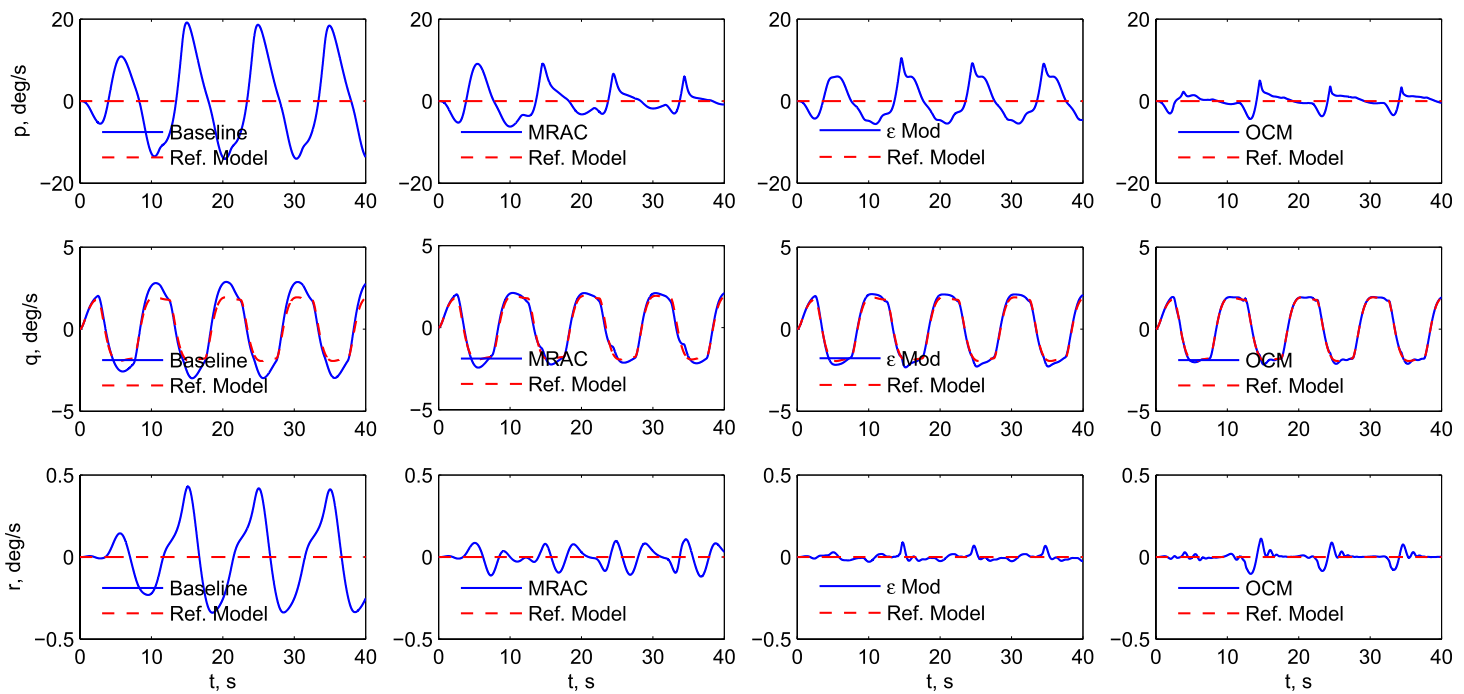

Fig. 3. Roll, pitch, and yaw rate responses.
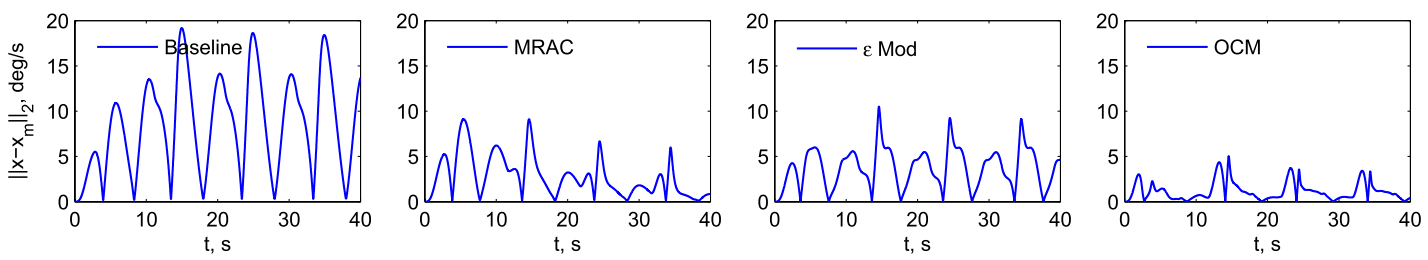

Fig. 4. Tracking error norm.
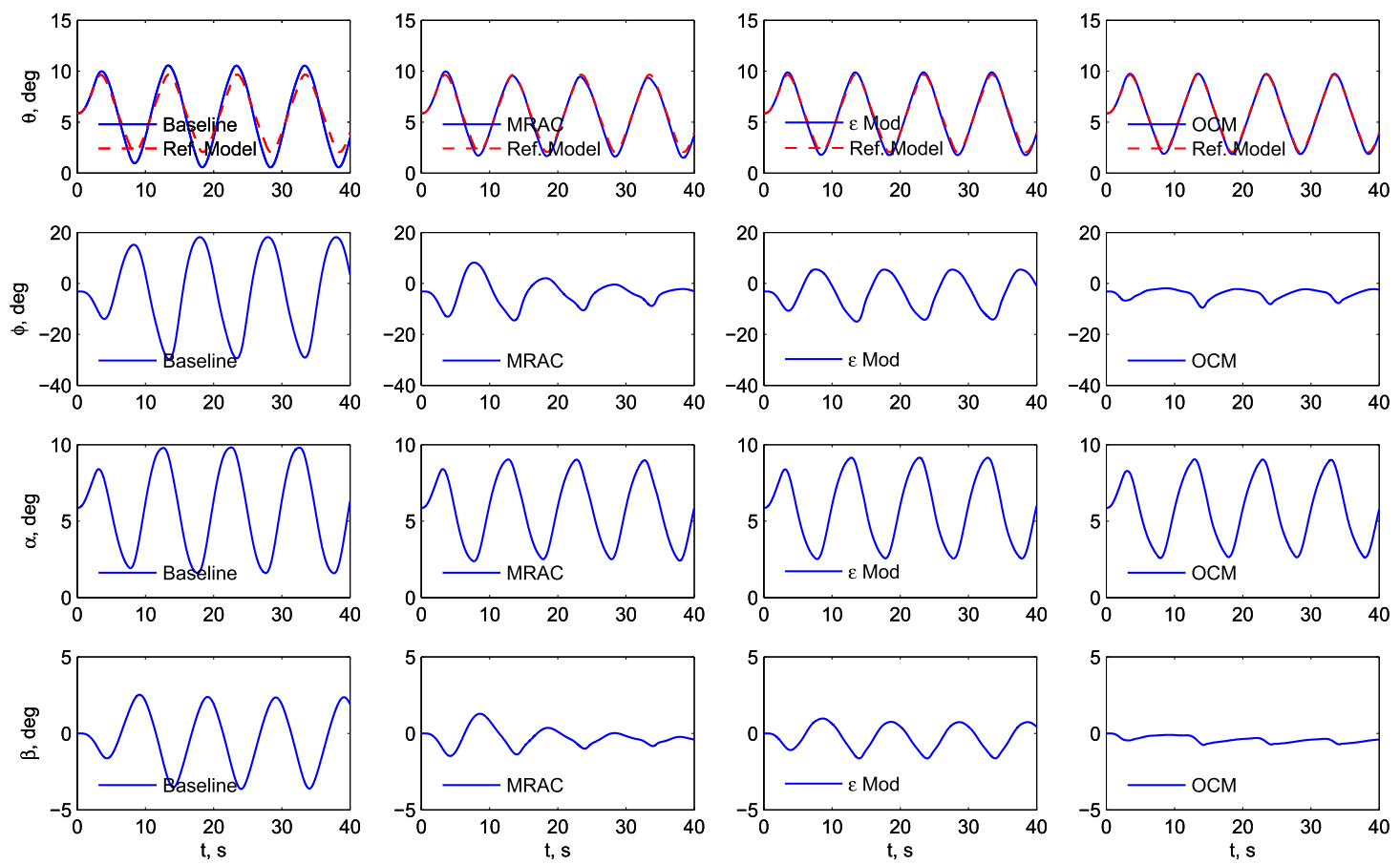

Fig. 5. Pitch angle, bank angle, angle of attack, and sideslip angle.

controller. On the other hand, all things being equal, increasing $v$ tends to degrade the tracking performance. Therefore, in general, $v$ is selected to balance the competing requirements for performance and robustness that usually exist in a control design.

\section{Pilot evaluation and flight testing}

The Integrated Resilient Aircraft Control (IRAC) project under the NASA Aviation Safety program from 2005 to 2010 conducted 

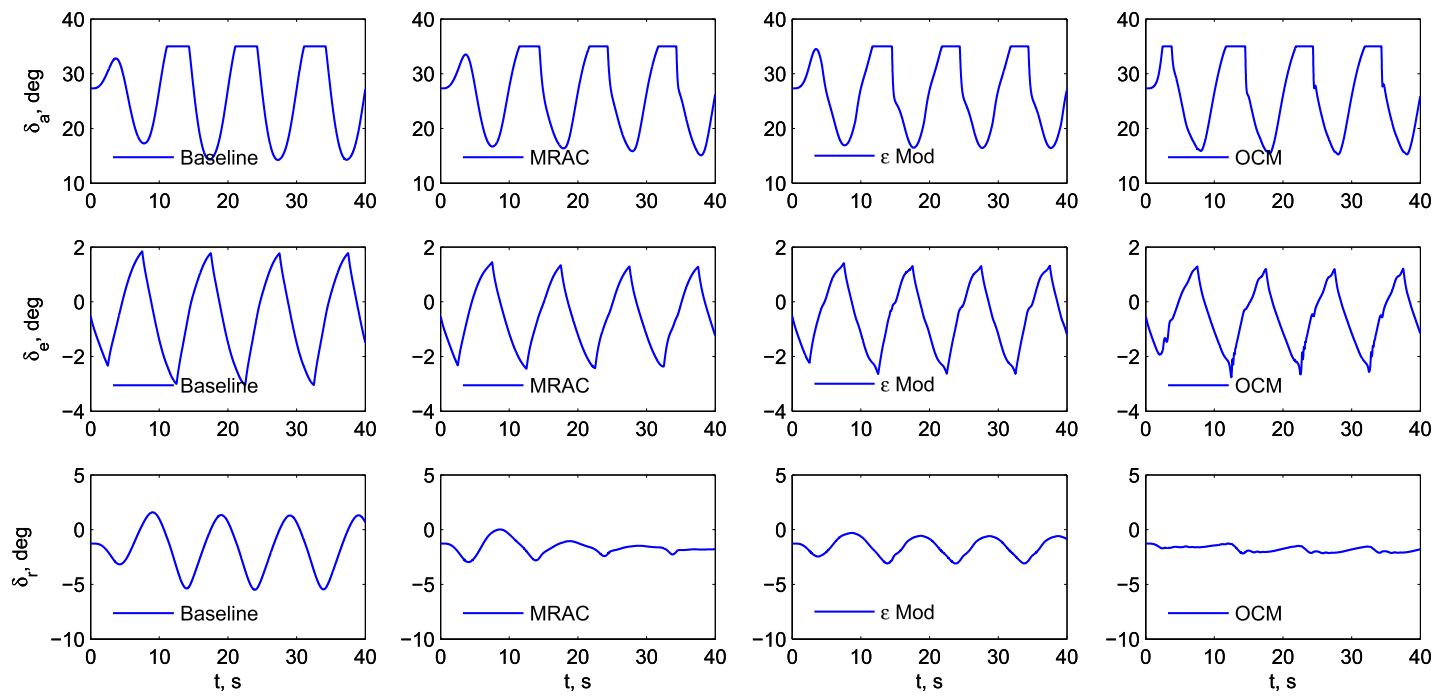

Fig. 6. Aileron, elevator, and rudder deflections

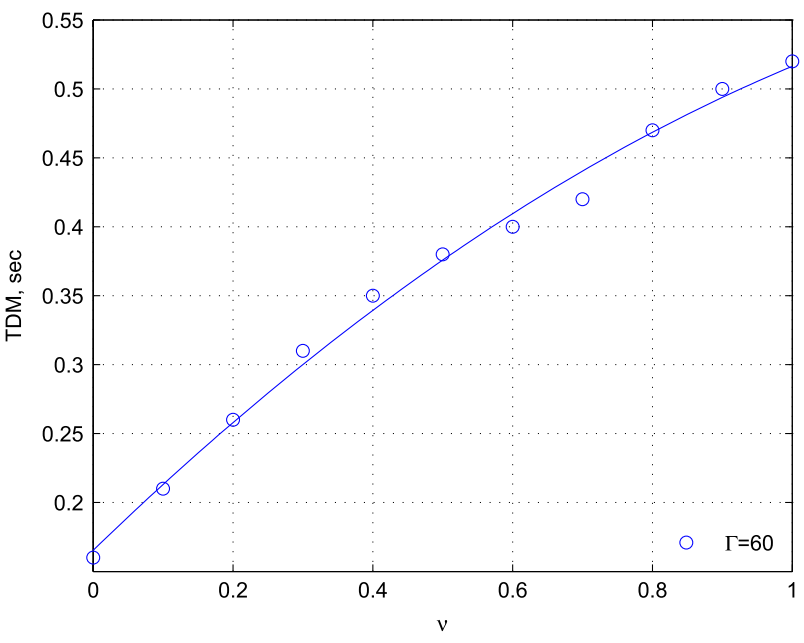

Fig. 7. Estimated time delay margin.

state-of-the-arts flight control research to provide on-board control resilience for ensuring flight safety in the presence of adverse flight conditions such as faults, damage, and/or upsets [18]. Maintaining aircraft stability and maneuverability in off-nominal flight conditions is critical to aircraft flight safety. Adaptive flight control is identified as an important technology that can improve aircraft stability and maneuverability, and thus was a key focus research area under the IRAC project. To advance the state-of-thearts adaptive control technology, the IRAC project developed plans to validate adaptive control at various levels of fidelity ranging from simple single-axis flight control simulations to fully nonlinear simulations in a pilot-in-the-loop flight simulator and ultimately to flight testing with a full-scale piloted aircraft.

In 2010, a pilot evaluation study was conducted in the Advanced Concept Flight Simulator (ACFS) at NASA Ames Research Center to evaluate a number of adaptive control methods including the optimal control modification adaptive law $[19,20]$. The ACFS, as shown in Fig. 8, is a motion-based flight simulator that has a highly customizable flight simulation environment which can be used to simulate a wide variety of transport-type aircraft. The ACFS employs advanced fly-by-wire digital flight control systems, a head-up display in the flight deck, a customizable flight management system, and modern flight instruments and electronics. Pilot inputs are provided by a side stick for controlling aircraft in pitch and roll axes.

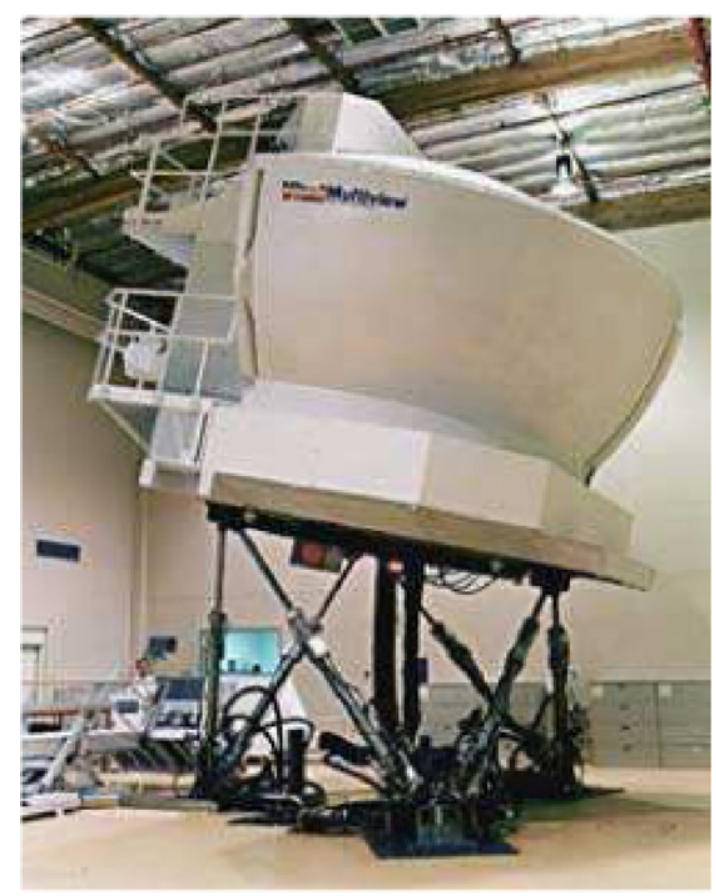

Fig. 8. NASA Advanced Concept Flight Simulator (ACFS).

For the pilot evaluation study, the ACFS was configured as the GTM. Failure and damage emulation included asymmetric damage to the left horizontal tail and elevator, flight control faults emulated by scaling the control sensitivity matrix ( $B$-matrix failures), and combined failures. Eight different NASA test pilots participated in the study. For each emulated failure, each of the pilots evaluated handling qualities by providing the Cooper-Harper ratings for a series of flight tasks, which included large amplitude attitude capture tasks and cross-wind approach and landing tasks. The results of the pilot evaluation study indicated that the optimal control modification performs quite well and provides consistent handling qualities under all different types of emulated failures and over all different flight conditions [20]. These results formed the basis for selecting the optimal control modification for flight testing.

The NASA Full-Scale Advanced Systems Testbed (FAST) is an F/A-18A research aircraft at NASA Dryden Flight Research Center as 


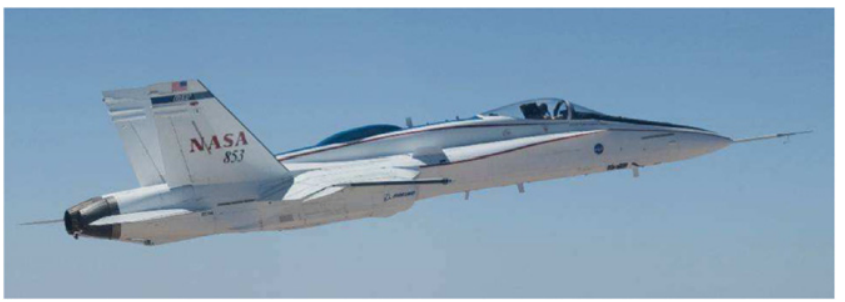

Fig. 9. NASA Full-Scale Advanced Systems Testbed (FAST).

shown in Fig. 9. The aircraft underwent an avionic system upgrade which includes a new ARTS (Airborne Research Test System) flight control computer system. The ARTS is capable of housing advanced flight control experiments. Failure emulations can be introduced via parameter changes in the flight control software to intentionally degrade aircraft stability, and through hardware emulated faults (frozen stabilator and cross-coupled pilot stick inputs) that cause cross-coupling in both the pitch and roll axes. Flight testing of adaptive control using a piloted, full-scale testbed has been shown to uncover implementation issues that may not be found through experiments with flight simulators or sub-scale, remotely piloted aircraft [21].

Prior to the flight control implementation on the aircraft, the optimal control modification was implemented in a high-fidelity flight dynamic model of the F/A-18A aircraft to evaluate the effectiveness of the adaptive law. The results show improvements in the tracking performance of the optimal control modification [22].

Three adaptive flight controllers were implemented for flight experiments. The first controller is based on a simplified standard MRAC (sMRAC) where the input to the adaptive law is simply a state variable which could be a pitch rate or a roll rate. The second controller is based on the optimal control modification adaptive law with normalization (onMRAC) [23]. The third controller is a variance of the second controller plus an additional optimal control modification term with a bias input (onMRAC+) to handle disturbances either due to input uncertainties such as failed control surfaces or external disturbances such as cross-coupling.

During a period from December of 2010 to January of 2011 , a series of flight test experiments of the three adaptive flight controllers were successfully demonstrated on board the F/A-18A aircraft at NASA Dryden Flight Research Center. Initial flight test results demonstrated an obvious improvement in-flight performance with the three controllers. The "onMRAC" was noted to be working quite well and appeared to adapt more quickly than "sMRAC" for the reduced pitch damping failure. Additionally, the adaptive law for the disturbance estimate in the "onMRAC+" configuration was found to exhibit small, persistent oscillations in the pitch axis. Between flights, an optimal control modification term was added to this update law and shown to effectively eliminate the objectionable oscillations. The results of flight testing have recently been published [21,24].

\section{Conclusions}

This study presents a new robust modification to the standard model-reference adaptive control based on an optimal control formulation of minimizing the $\mathscr{L}_{2}$ norm of the tracking error. The modification enables fast adaptation for improving tracking performance without sacrificing stability robustness. The optimal control modification adaptive law can be tuned using a modification parameter $v$ to provide a trade-off between tracking performance and stability robustness. Increasing $v$ improves robustness to time delay and or unmodeled dynamics but reduces tracking performance. Simulation results of a damaged generic transport aircraft demonstrate the effectiveness of the optimal control modification adaptive law, which improves the tracking performance significantly using a much larger adaptive gain than that for the standard model-reference adaptive control. Pilot evaluation study in a motion-based flight simulator at NASA Ames Research Center confirms the effectiveness of the optimal control modification. A series of recent, successful flight experiments of this adaptive law on a NASA F/A-18A aircraft at NASA Dryden Flight Research Center further demonstrate the potential benefits of the optimal control modification.

\section{Acknowledgments}

The author would like to thank the reviewers for their constructive inputs in their reviews and valuable contributions in improving the manuscript. The author also would like to acknowledge NASA Aeronautics Research Mission Directorate Aviation Safety Program for providing support of this research.

\section{References}

[1] J. Bosworth, P.S. Williams-Hayes, Flight test results from the NF-15B IFCS project with adaptation to a simulated stabilator failure AIAA Infotech@Aerospace Conference, AIAA-2007-2818, May 2007.

[2] N. Nguyen, S. Jacklin, Stability, convergence, and verification and validation challenges of neural net adaptive flight control, in: Applications of Neural Networks in High Assurance Systems Studies in Computational Intelligence, vol. 268/2010, Springer-Verlag, 2010, pp. 77-110.

[3] P.A. Ioannu, J. Sun, Robust Adaptive Control, Prentice-Hall, Inc., 1996.

[4] C. Cao, N. Hovakimyan, Design and analysis of a novel $\mathscr{L}_{1}$ adaptive control architecture with guaranteed transient performance, IEEE Transactions on Automatic Control 53 (2) (2008) 586-591.

[5] N. Nguyen, K. Krishnakumar, J. Kaneshige, P. Nespeca, Flight dynamics and hybrid adaptive control of damaged aircraft, AIAA Journal of Guidance, Control, and Dynamics 31 (3) (2008) 751-764.

[6] P. Ioannou, P. Kokotovic, Instability analysis and improvement of robustness of adaptive control, Automatica 20 (5) (1984) 583-594.

[7] K.S. Narendra, A.M. Annaswamy, A new adaptive law for robust adaptation without persistent excitation, IEEE Transactions on Automatic Control AC-32 (2) (1987) 134-145.

[8] N. Nguyen, K. Krishnakumar, J. Boskovic, An optimal control modification to model reference adaptive control for fast adaptation, in: AIAA Guidance, Navigation, and Control Conference, AIAA-2008-7283, August 2008.

[9] G. Cybenko, Approximation by superpositions of a sigmoidal function, Mathematics of Control Signals Systems 2 (1989) 303-314.

[10] C.A. Micchelli, Interpolation of scattered data: distance matrices and conditionally positive definite functions, Constructive Approximation 2 (1986) 11-12.

[11] N. Nguyen, J. Burken, A. Ishihara, Least-squares adaptive control using chevbyshev orthogonal polynomials, in: AIAA Infotech@Aerospace Conference, AIAA-2011-1402, March 2011.

[12] A.E. Bryson, Y.C. Ho, Applied Optimal Control: Optimization, Estimation, and Control, John Wiley \& Sons, Inc., 1979.

[13] B. Anderson, J. Moore, Linear Optimal Control, Prentice-Hall, Inc., 1971.

[14] C.E. Rohrs, L. Valavani, M. Athans, G. Stein, Robustness of continuous-time adaptive control algorithms in the presence of unmodeled dynamics, IEEE Transactions on Automatic Control AC-30 (9) (1985) 881-889.

[15] J.-J. Slotine, W. Li, Applied Nonlinear Control, Prentice-Hall, Inc., 1991.

[16] A. Calise, T. Yucelen, J. Muse, B. Yang, A loop recovery method for adaptive control, AIAA Guidance, Navigation, and Control Conference, AIAA-2009-5967, 2009.

[17] T. Jordan, W. Langford, C. Belcastro, J. Foster, G. Shah, G. Howland, R. Kidd, Development of a dynamically scaled generic transport model testbed for flight research experiments, in: AUVSI Unmanned Unlimited, Arlington, VA, 2004.

[18] K. Krishnakumar, S. Vikien, N. Nguyen, Integrated resilient aircraft controlstability, maneuverability, and safe landing in the presence of adverse conditions, NASA Aeronautics Research Mission Directorate Aviation Safety Program (May 1, 2009).

[19] S. Campbell, J. Kaneshige, N. Nguyen, K. Krishnakumar, Implementation and evaluation of multiple adaptive control technologies for a generic transport aircraft simulation, in: AIAA Infotech@Aerospace Conference, AIAA-20103322, April 2010.

[20] S. Campbell, J. Kaneshige, N. Nguyen, K. Krishnakumar, An adaptive control simulation study using metrics and pilot handling qualities evaluations, in: AIAA Guidance, Navigation, and Control Conference, AIAA-2010-8013, August 2010. 
[21] C. Hanson, M. Johnson, J. Schaefer, N. Nguyen, J. Burken, Handling qualities evaluations of low complexity model reference adaptive controllers for reduced pitch and roll damping scenarios, in: AIAA Guidance, Navigation, and Control Conference, AIAA-2011-6607, August 2011.

[22] J. Burken, N. Nguyen, B. Griffin, Adaptive flight control design with optimal control modification for F-18 aircraft model, in: AIAA Infotech@Aerospace Conference, AIAA-2010-3364, April 2010
[23] N. Nguyen, J. Burken, C. Hanson, Optimal control modification adaptive law with covariance adaptive gain adjustment and normalization, in: AIAA Guidance, Navigation, and Control Conference, AIAA-2011-6606, August 2011

[24] J. Schaefer, C. Hanson, M. Johnson, N. Nguyen, Handling qualities of mode reference adaptive controllers with varying complexity for pitch-roll coupled failures, in: AIAA Guidance, Navigation, and Control Conference, AIAA-20116453, August 2011. 